\title{
Positive solutions to nonlinear first-order impulsive dynamic equations on time scales
}

\section{Wen Guan*}

\section{"Correspondence:}

mathguanw@163.com

Department of Applied

Mathematics, Lanzhou University of

Technology, Lanzhou, Gansu

730050, People's Republic of China

\begin{abstract}
By using the classical fixed point theorem for operators on a cone, in this paper, some results of single and multiple positive solutions to a class of nonlinear first-order periodic boundary value problems of impulsive dynamic equations on time scales are obtained. It is worth noticing that the nonlinearity $f$ and the pulse function in this paper are not positive.
\end{abstract}

MSC: 39A10; 34B15

Keywords: time scale; periodic boundary value problem; fixed point; impulsive dynamic equation

\section{Introduction}

The theory of dynamic equations on time scales has been a new important mathematical branch [1-3] since it was initiated by Hilger [4]. At the same time, the boundary value problems of impulsive dynamic equations on time scales have received considerable attention [5-21] since the theory of impulsive differential equations is much richer than the corresponding theory of differential equations without impulse effects [22-24].

In [18], by using the Guo-Krasnoselskii fixed point theorem, when the nonlinearity $f$ and the pulse function are positive, Wang considered the existence of one or two positive solutions to the following PBVPs of impulsive dynamic equations on time scales:

$$
\left\{\begin{array}{l}
x^{\Delta}(t)+p(t) x(\sigma(t))=f(t, x(\sigma(t))), \quad t \in J:=[0, T]_{\mathbb{T}}, t \neq t_{k}, \\
x\left(t_{k}^{+}\right)-x\left(t_{k}^{-}\right)=I_{k}\left(x\left(t_{k}^{-}\right)\right), \quad k=1,2, \ldots, m, \\
x(0)=x(\sigma(T)) .
\end{array}\right.
$$

In [20], by using the Schaefer fixed point theorem, Wang and Weng obtained the existence of at least one solution to the problem (1.1).

Motivated by the results mentioned above, in this paper, we shall obtain the existence of single and multiplicity positive solutions to the problem (1.1) where $\mathbb{T}$ is an arbitrary time scale, $T>0$ is fixed, $0, T \in \mathbb{T}, f \in C(J \times[0, \infty), \mathbb{R}), I_{k} \in C([0, \infty), \mathbb{R}), p:[0, T]_{\mathbb{T}} \rightarrow(0, \infty)$ is right-dense continuous, $t_{k} \in(0, T)_{\mathbb{T}}, 0<t_{1}<\cdots<t_{m}<T$, and for each $k=1,2, \ldots, m$, $x\left(t_{k}^{+}\right)=\lim _{h \rightarrow 0^{+}} x\left(t_{k}+h\right)$ and $x\left(t_{k}^{-}\right)=\lim _{h \rightarrow 0^{-}} x\left(t_{k}+h\right)$ which represent the right and left limits of $x(t)$ at $t=t_{k}$. The main tool used in this paper is the classical fixed point theorem for operators on a cone.

( 2015 Guan; licensee Springer. This is an Open Access article distributed under the terms of the Creative Commons Attribution License (http://creativecommons.org/licenses/by/4.0), which permits unrestricted use, distribution, and reproduction in any medium, provided the original work is properly credited. 
It is worth noticing that: (i) The nonlinearity $f$ and the pulse function in this paper are not positive, so our hypotheses on the nonlinearity $f$ and the pulse function are weaker than condition of [18]. (ii) For the case $I_{k}(x) \equiv 0, k=1,2, \ldots, m$, the problem (1.1) reduces to the problem studied by [25, 26]; for the case $p(t)=0$, the problem (1.1) reduces to the problem studied by $[12,19]$; for the case $p(t)=0$ and $\mathbb{T}=\mathbb{Z}$, the problem (1.1) reduces to the problem studied by [27]. This paper's ideas come from [28].

Throughout this work, we assume the knowledge of time scales and time-scale notation, first introduced by Hilger [4]. For more information on time scales, please see the texts by Bohner and Peterson $[2,3]$.

In the remainder of this section, we state the following fixed point theorem [29].

Theorem 1.1 [29] Let $X$ be a Banach space and $K$ is a cone in $X$. Assume $\Omega_{1}, \Omega_{2}$ are open subsets of $X$ with $0 \in \Omega_{1}, \bar{\Omega}_{1} \subset \Omega_{2}$. Let

$$
\Phi: K \cap\left(\bar{\Omega}_{2} \backslash \Omega_{1}\right) \rightarrow K
$$

be a continuous and completely continuous operator such that

(i) $\|\Phi x\| \leq\|x\|$ for $x \in K \cap \partial \Omega_{1}$;

(ii) there exists $e \in K \backslash\{0\}$ such that $x \neq \Phi x+\lambda$ efor $x \in K \cap \partial \Omega_{2}$ and $\lambda>0$.

Then $\Phi$ has a fixed point in $K \cap\left(\bar{\Omega}_{2} \backslash \Omega_{1}\right)$.

Remark 1.1 In Theorem 1.1, if (i) and (ii) are replaced by

(i) $\|\Phi x\| \leq\|x\|$ for $x \in K \cap \partial \Omega_{2}$;

(ii) there exists $e \in K \backslash\{0\}$ such that $x \neq \Phi x+\lambda e$ for $x \in K \cap \partial \Omega_{1}$ and $\lambda>0$, then $\Phi$ has also a fixed point in $K \cap\left(\bar{\Omega}_{2} \backslash \Omega_{1}\right)$.

\section{Main results}

Throughout the rest of this paper, we always assume that the points of impulse $t_{k}$ are rightdense for each $k=1,2, \ldots, m$.

We define

$$
\begin{aligned}
P C= & \left\{x \in[0, \sigma(T)]_{\mathbb{T}} \rightarrow \mathbb{R}: x_{k} \in C\left(J_{k}, \mathbb{R}\right), k=0,1,2, \ldots, m,\right. \text { and there exist } \\
& \left.x\left(t_{k}^{+}\right) \text {and } x\left(t_{k}^{-}\right) \text {with } x\left(t_{k}^{-}\right)=x\left(t_{k}\right), k=1,2, \ldots, m\right\},
\end{aligned}
$$

where $x_{k}$ is the restriction of $x$ to $J_{k}=\left(t_{k}, t_{k+1}\right]_{\mathbb{T}} \subset(0, \sigma(T)]_{\mathbb{T}}, k=1,2, \ldots, m$, and $J_{0}=\left[0, t_{1}\right]_{\mathbb{T}}$, $t_{m+1}=\sigma(T)$.

Let

$$
X=\{x: x \in P C, x(0)=x(\sigma(T))\}
$$

with the norm $\|x\|=\sup _{t \in[0, \sigma(T)]_{\mathbb{T}}}|x(t)|$, then $X$ is a Banach space.

Lemma 2.1 [18] Suppose $h:[0, T]_{\mathbb{T}} \rightarrow \mathbb{R}$ is $r d$-continuous, then $x$ is a solution of

$$
x(t)=\int_{0}^{\sigma(T)} G(t, s) h(s) \Delta s+\sum_{k=1}^{m} G\left(t, t_{k}\right) I_{k}\left(x\left(t_{k}\right)\right), \quad t \in[0, \sigma(T)]_{\mathbb{T}},
$$


where

$$
G(t, s)= \begin{cases}\frac{e_{p}(s, t) e_{p}(\sigma(T), 0)}{e_{p}(\sigma(T), 0)-1}, & 0 \leq s \leq t \leq \sigma(T), \\ \frac{e_{p}(s, t)}{e_{p}(\sigma(T), 0)-1}, & 0 \leq t<s \leq \sigma(T),\end{cases}
$$

if and only if $x$ is a solution of the boundary value problem

$$
\left\{\begin{array}{l}
x^{\Delta}(t)+p(t) x(\sigma(t))=h(t), \quad t \in J, t \neq t_{k}, \\
x\left(t_{k}^{+}\right)-x\left(t_{k}^{-}\right)=I_{k}\left(x\left(t_{k}^{-}\right)\right), \quad k=1,2, \ldots, m, \\
x(0)=x(\sigma(T)) .
\end{array}\right.
$$

Lemma 2.2 Let $G(t, s)$ be defined as in Lemma 2.1, then

$$
A=\frac{1}{e_{p}(\sigma(T), 0)-1} \leq G(t, s) \leq \frac{e_{p}(\sigma(T), 0)}{e_{p}(\sigma(T), 0)-1}=B \quad \text { for all } t, s \in[0, \sigma(T)]_{\mathbb{T}} .
$$

Remark 2.1 Let $G(t, s)$ be defined as in Lemma 2.1, then $\int_{0}^{\sigma(T)} G(t, s) p(s) \triangle s=1$.

Let

$$
K=\left\{x \in X: x(t) \geq \delta\|x\|, t \in[0, \sigma(T)]_{\mathbb{T}}\right\},
$$

where $\delta=\frac{A}{B} \in(0,1)$. It is not difficult to verify that $K$ is a cone in $X$.

For convenience, we denote

$$
\begin{array}{ll}
f^{0}=\lim _{x \rightarrow 0^{+}} \sup _{t \in[0, T]_{\mathbb{T}}} \frac{f(t, x)}{p(t) x}, & I^{0}(k)=\lim _{x \rightarrow 0^{+}} \sup \frac{I_{k}(x)}{x} ; \\
f_{0}=\lim _{x \rightarrow 0^{+}} \inf \min _{t \in[0, T]_{\mathbb{T}}} \frac{f(t, x)}{p(t) x}, & I_{0}(k)=\lim _{x \rightarrow 0^{+}} \inf \frac{I_{k}(x)}{x} ; \\
f^{\infty}=\lim _{x \rightarrow \infty} \sup _{f_{t \in[0, T]_{\mathbb{T}}} \frac{f(t, x)}{p(t) x},}=\lim _{x \rightarrow \infty} \inf _{t \in[0, T]_{\mathbb{T}}} \frac{f(t, x)}{p(t) x}, & I_{\infty}(k)=\lim _{x \rightarrow \infty} \sup \frac{I_{k}(x)}{x} ; \\
f^{u}=\sup _{x \in[\delta u, u]} \max _{t \in[0, T]_{\mathbb{T}}} \frac{f(t, x)}{p(t) x}, & I^{u}(k)=\sup _{x \in[\delta u, u]} \frac{I_{k}(x)}{x} ; \\
f_{u}=\inf _{x \in[\delta u, u]} \min _{t \in[0, T]_{\mathbb{T}}} \frac{f(t, x)}{p(t) x}, & I_{u}(k)=\inf _{x \in[\delta u, u]} \frac{I_{k}(x)}{x} .
\end{array}
$$

Now we state our main results.

Theorem 2.1 Suppose there exist $0<\alpha<\beta$ such that

$$
f(t, x) \geq 0 \quad \text { and } \quad I_{k}(x) \geq 0, \quad t \in[0, T]_{\mathbb{T}}, x \in[\delta \alpha, \beta] .
$$

Then the problem (1.1) has at least one positive solution if one of the following two conditions holds:

(i) $f_{\alpha}+A \sum_{k=1}^{m} I_{\alpha}(k) \geq 1, f^{\beta}+B \sum_{k=1}^{m} I^{\beta}(k) \leq 1$;

(ii) $f^{\alpha}+B \sum_{k=1}^{m} I^{\alpha}(k) \leq 1, f_{\beta}+A \sum_{k=1}^{m} I_{\beta}(k) \geq 1$. 
Proof We define an operator $\Phi: K \rightarrow X$ by

$$
\Phi(x)(t)=\int_{0}^{\sigma(T)} G(t, s) f(s, x(\sigma(s))) \triangle s+\sum_{k=1}^{m} G\left(t, t_{k}\right) I_{k}\left(u\left(t_{k}\right)\right), \quad t \in[0, \sigma(T)]_{\mathbb{T}} .
$$

It is obvious that fixed points of $\Phi$ are solutions of the problem (1.1). Similar to [18], $\Phi: K \rightarrow X$ is completely continuous.

Define the open sets

$$
\begin{aligned}
& \Omega_{1}=\{x \in X:\|x\|<\alpha\}, \\
& \Omega_{2}=\{x \in X:\|x\|<\beta\} .
\end{aligned}
$$

Firstly, we claim that $\Phi: K \cap\left(\bar{\Omega}_{2} \backslash \Omega_{1}\right) \rightarrow K$.

In fact, for any $x \in K \cap\left(\bar{\Omega}_{2} \backslash \Omega_{1}\right)$, we have $\delta \alpha \leq x \leq \beta$, by Lemma 2.2

$$
\|\Phi x\| \leq B\left[\int_{0}^{\sigma(T)} f(s, x(\sigma(s))) \Delta s+\sum_{k=1}^{m} I_{k}\left(x\left(t_{k}\right)\right)\right]
$$

and

$$
\begin{aligned}
(\Phi x)(t) & =\int_{0}^{\sigma(T)} G(t, s) h_{x}(s) \triangle s+\sum_{k=1}^{m} G\left(t, t_{k}\right) I_{k}\left(x\left(t_{k}\right)\right) \\
& \geq A\left[\int_{0}^{\sigma(T)} f(s, x(\sigma(s))) \Delta s+\sum_{k=1}^{m} I_{k}\left(x\left(t_{k}\right)\right)\right] .
\end{aligned}
$$

So

$$
(\Phi x)(t) \geq \frac{A}{B}\|\Phi x\|=\delta\|\Phi x\|, \quad \text { i.e., } \Phi x \in K .
$$

Therefore, $\Phi: K \cap\left(\bar{\Omega}_{2} \backslash \Omega_{1}\right) \rightarrow K$.

Secondly, we prove the result provided condition (i) holds.

Since

$$
f(t, x) \geq f_{\alpha} p(t) x, \quad I_{k}(x) \geq I_{\alpha}(k) x, \quad k=1,2, \ldots, m, x \in[\delta \alpha, \alpha] .
$$

Let $e \equiv 1$, then $e \in K$. We assert that

$$
x \neq \Phi x+\lambda e \quad \text { for } x \in K \cap \partial \Omega_{1} \text { and } \lambda>0 .
$$

If not, there would exist $x_{0} \in K \cap \partial \Omega_{1}$ and $\lambda_{0}>0$ such that $x_{0}=\Phi x_{0}+\lambda_{0} e$.

Since $x_{0} \in K \cap \partial \Omega_{1}$, then $\delta \alpha=\delta\left\|x_{0}\right\| \leq x_{0}(t) \leq \alpha$. Let $\mu=\min _{t \in[0, \sigma(T)]_{\mathbb{T}}} x_{0}(t)$, then for any $t \in[0, \sigma(T)]_{\mathbb{T}}$, by the first inequality of (i) we have

$$
\begin{aligned}
x_{0}(t) & =\left(\Phi x_{0}\right)(t)+\lambda_{0} \\
& =\int_{0}^{\sigma(T)} G(t, s) f\left(s, x_{0}(\sigma(s))\right) \triangle s+\sum_{k=1}^{m} G\left(t, t_{k}\right) I_{k}\left(x_{0}\left(t_{k}\right)\right)+\lambda_{0}
\end{aligned}
$$




$$
\begin{aligned}
& \geq \int_{0}^{\sigma(T)} G(t, s) f_{\alpha} p(s) x_{0}(\sigma(s)) \triangle s+A \sum_{k=1}^{m} I_{\alpha}(k) x\left(t_{k}\right)+\lambda_{0} \\
& \geq \mu\left[f_{\alpha}+A \sum_{k=1}^{m} I_{\alpha}(k)\right]+\lambda_{0} \\
& \geq \mu+\lambda_{0} .
\end{aligned}
$$

This implies that $\mu \geq \mu+\lambda_{0}$, and this is a contradiction. Therefore (2.1) holds.

On the other hand, by using the second inequality of (i), we have

$$
f(t, x) \leq\left[1-B \sum_{k=1}^{m} I^{\beta}(k)\right] p(t) x, \quad t \in[0, T]_{\mathbb{T}}, x \in[\delta \beta, \beta] .
$$

We assert that

$$
\|\Phi x\| \leq\|x\| \quad \text { for } x \in K \cap \partial \Omega_{2}
$$

In fact, for any $x \in K \cap \partial \Omega_{2}$, then $\delta \beta=\delta\|x\| \leq x(t) \leq \beta$, we have

$$
\begin{aligned}
(\Phi x)(t) & =\int_{0}^{\sigma(T)} G(t, s) f(s, x(\sigma(s))) \triangle s+\sum_{k=1}^{m} G\left(t, t_{k}\right) I_{k}\left(x\left(t_{k}\right)\right) \\
& \leq\left(1-B \sum_{k=1}^{m} I^{\beta}(k)\right) \int_{0}^{\sigma(T)} G(t, s) p(s) x(\sigma(s)) \triangle s+B \sum_{k=1}^{m} I^{\beta}(k) x\left(t_{k}\right) \\
& \leq\left[\left(1-B \sum_{k=1}^{m} I^{\beta}(k)\right) \int_{0}^{\sigma(T)} G(t, s) p(s) \triangle s+B \sum_{k=1}^{m} I^{\beta}(k)\right]\|x\| \\
& =\|x\| .
\end{aligned}
$$

Therefore, $\|\Phi x\| \leq\|x\|$.

It follows from Remark 1.1, (2.1), and (2.2) that $\Phi$ has a fixed point $x \in K \cap\left(\bar{\Omega}_{2} \backslash \Omega_{1}\right)$. In a similar way, we can prove the result by Theorem 1.1 if condition (ii) holds.

Theorem 2.2 Suppose there exist $0<\alpha<\rho<\beta$ such that

$$
f(t, x) \geq 0 \quad \text { and } \quad I_{k}(x) \geq 0, \quad t \in[0, T]_{\mathbb{T}}, x \in[\delta \alpha, \beta]
$$

Then the problem (1.1) has at least two positive solution if one of the following two conditions holds:

(i) $f_{\alpha}+A \sum_{k=1}^{m} I_{\alpha}(k) \geq 1, f^{\rho}+B \sum_{k=1}^{m} I^{\rho}(k)<1, f_{\beta}+A \sum_{k=1}^{m} I_{\beta}(k) \geq 1$;

(ii) $f^{\alpha}+B \sum_{k=1}^{m} I^{\alpha}(k) \leq 1, f_{\rho}+A \sum_{k=1}^{m} I_{\rho}(k)>1, f^{\beta}+B \sum_{k=1}^{m} I^{\beta}(k) \leq 1$.

Proof We only prove the result when condition (i) holds. In a similar way we can obtain the result if condition (ii) holds.

Define $\Omega_{1}, \Omega_{2}$ as in Theorem 2.1 and define

$$
\Omega_{3}=\{x \in X:\|x\|<\rho\} .
$$


Similar to the proof of Theorem 2.1, we can prove that

$$
\begin{array}{ll}
x \neq \Phi x+\lambda e & \text { for } x \in K \cap \partial \Omega_{1} \text { and } \lambda>0, \\
x \neq \Phi x+\lambda e & \text { for } x \in K \cap \partial \Omega_{2} \text { and } \lambda>0,
\end{array}
$$

where $e \equiv 1 \in K$, and

$$
\|\Phi x\|<\|x\| \quad \text { for } x \in K \cap \partial \Omega_{3} .
$$

Thus we can obtain the existence of two positive solutions $x_{1}$ and $x_{2}$ by using Theorem 1.1 and Remark 1.1, respectively. It is easy to see that $\alpha \leq\left\|x_{1}\right\|<\rho<\left\|x_{2}\right\| \leq \beta$.

Theorem 2.3 Suppose that there exist positive $0<\alpha_{1}<\beta_{1}<\alpha_{2}<\beta_{2}<\cdots<\alpha_{n}<\beta_{n}$ such that

$$
f(t, x) \geq 0 \quad \text { and } \quad I_{k}(x) \geq 0, \quad t \in[0, T]_{\mathbb{T}}, x \in\left[\delta \alpha_{1}, \beta_{n}\right]
$$

Then the problem (1.1) has at least $n$ positive solutions $x_{i}(1 \leq i \leq n)$ satisfying $\alpha_{i} \leq\left\|x_{i}\right\| \leq$ $\beta_{i}, 1 \leq i \leq n$, if one of the following two conditions holds:

(i) $f_{\alpha_{i}}+A \sum_{k=1}^{m} I_{\alpha_{i}}(k) \geq 1, f^{\beta_{i}}+B \sum_{k=1}^{m} I^{\beta_{i}}(k) \leq 1$;

(ii) $f^{\alpha_{i}}+B \sum_{k=1}^{m} I^{\alpha_{i}}(k) \leq 1, f_{\beta_{i}}+A \sum_{k=1}^{m} I_{\beta_{i}}(k) \geq 1$.

Remark 2.2 In Theorem 2.3, assume (i) and (ii) are replaced by

(i) $f_{\alpha_{i}}+A \sum_{k=1}^{m} I_{\alpha_{i}}(k)>1, f^{\beta_{i}}+B \sum_{k=1}^{m} I^{\beta_{i}}(k)<1$;

(ii) $f^{\alpha_{i}}+B \sum_{k=1}^{m} I^{\alpha_{i}}(k)<1, f_{\beta_{i}}+A \sum_{k=1}^{m} I_{\beta_{i}}(k)>1$.

Then the problem (1.1) has at least $2 n-1$ positive solutions.

\section{Application}

In this section, we are going to apply our main existence results obtained in Section 2 to some illustrating examples.

Theorem 3.1 Suppose that $f(t, x) \geq 0$ and $I_{k}(x) \geq 0, t \in[0, T]_{\mathbb{T}}, x \in \mathbb{R}^{+}=[0, \infty)$. Then the problem (1.1) has at least one positive solution if one of the following two conditions holds:

(i) $f_{0}+A \sum_{k=1}^{m} I_{0}(k)>1, f^{\infty}+B \sum_{k=1}^{m} I^{\infty}(k)<1$;

(ii) $f^{0}+B \sum_{k=1}^{m} I^{0}(k)<1, f_{\infty}+A \sum_{k=1}^{m} I_{\infty}(k)>1$.

Proof It is a direct consequence of Theorem 2.1 taking $\alpha$ small enough and $\beta$ large enough, respectively.

In particular, we have the following results, the main results of [18].

Corollary 3.1 Suppose that $f(t, x) \geq 0$ and $I_{k}(x) \geq 0, t \in[0, T]_{\mathbb{T}}, x \in \mathbb{R}^{+}$. Then the problem (1.1) has at least one positive solution if one of the following two conditions holds:

(i) $f_{0}=\infty$ or $\sum_{k=1}^{m} I_{0}(k)=\infty, f^{\infty}=0, \sum_{k=1}^{m} I^{\infty}(k)=0$;

(ii) $f_{\infty}=\infty$ or $I_{\infty}(k)=\infty, f^{0}=0, \sum_{k=1}^{m} I^{0}(k)=0$. 
Example 3.1 We consider the following problem on $\mathbb{T}$ :

$$
\left\{\begin{array}{l}
x^{\triangle}(t)+p(t) x(\sigma(t))=(x(\sigma(t)))^{a}+(x(\sigma(t)))^{b}, \quad t \in J, t \neq t_{k}, \\
x\left(t_{k}^{+}\right)-x\left(t_{k}^{-}\right)=\left(x\left(t_{k}^{-}\right)\right)^{a^{\prime}}+\left(x\left(t_{k}^{-}\right)\right)^{b^{\prime}}, \quad k=1,2, \ldots, m, \\
x(0)=x(\sigma(T)),
\end{array}\right.
$$

where $p:[0, T]_{\mathbb{T}} \rightarrow(0, \infty)$ is right-dense continuous, $0<a, b<1,0<a^{\prime}, b^{\prime}<1$ or $a, b>1$, $a^{\prime}, b^{\prime}>1$.

Then it is easy to see that

$$
f_{0}=\infty, \quad \sum_{k=1}^{m} I_{0}(k)=\infty, \quad f^{\infty}=0, \quad \sum_{k=1}^{m} I^{\infty}(k)=0
$$

for $0<a, b<1,0<a^{\prime}, b^{\prime}<1$,

$$
f_{\infty}=\infty, \quad I_{\infty}(k)=\infty, \quad f^{0}=0, \quad \sum_{k=1}^{m} I^{0}(k)=0
$$

for $a, b>1, a^{\prime}, b^{\prime}>1$.

Therefore, by Corollary 3.1, it follows that the problem (3.1) has at least one positive solution.

Theorem 3.2 Suppose that $f(t, x) \geq 0$ and $I_{k}(x) \geq 0, t \in[0, T]_{\mathbb{T}}, x \in \mathbb{R}^{+}$. Then the problem (1.1) has at least two positive solutions if one of the following two conditions holds:

(i) $f_{0}+A \sum_{k=1}^{m} I_{0}(k)>1, f_{\infty}+A \sum_{k=1}^{m} I_{\infty}(k)>1$ and there exists $a \rho>0$ such that $\delta \rho \leq x \leq \rho$ implies

$$
f(t, x) / p(t) x+B \sum_{k=1}^{m} I^{\rho}(k)<1, \quad t \in[0, T]_{\mathbb{T}}
$$

(ii) $f^{0}+B \sum_{k=1}^{m} I^{0}(k)<1, f^{\infty}+B \sum_{k=1}^{m} I^{\infty}(k)<1$ and there exists a $\rho>0$ such that $\delta \rho \leq x \leq \rho$ implies

$$
f(t, x) / p(t) x+A \sum_{k=1}^{m} I_{\rho}(k)>1, \quad t \in[0, T]_{\mathbb{T}} .
$$

Corollary 3.2 Suppose that $f(t, x) \geq 0$ and $I_{k}(x) \geq 0, t \in[0, T]_{\mathbb{T}}, x \in \mathbb{R}^{+}$. Then the problem

(1.1) has at least two positive solutions if one of the following two conditions holds:

(i) $f_{0}=\infty$ or $\sum_{k=1}^{m} I_{0}(k)=\infty, f_{\infty}=\infty$ or $I_{\infty}(k)=\infty$, and there exists a $\rho>0$ such that $\delta \rho \leq x \leq \rho$ implies

$$
f(t, x) / p(t) x+B \sum_{k=1}^{m} I^{\rho}(k)<1, \quad t \in[0, T]_{\mathbb{T}} ;
$$

(ii) $f^{\infty}=0, \sum_{k=1}^{m} I^{\infty}(k)=0, f^{0}=0, \sum_{k=1}^{m} I^{0}(k)=0$, and there exists a $\rho>0$ such that $\delta \rho \leq x \leq \rho$ implies

$$
f(t, x) / p(t) x+A \sum_{k=1}^{m} I_{\rho}(k)>1, \quad t \in[0, T]_{\mathbb{T}} .
$$


Example 3.2 We consider the following problem on $\mathbb{T}$ :

$$
\left\{\begin{array}{l}
x^{\triangle}(t)+p(t) x(\sigma(t))=q(t)\left[(x(\sigma(t)))^{a}+(x(\sigma(t)))^{b}\right], \quad t \in J, t \neq t_{k} \\
x\left(t_{k}^{+}\right)-x\left(t_{k}^{-}\right)=a_{k} x\left(t_{k}^{-}\right), \quad k=1,2, \ldots, m, \\
x(0)=x(\sigma(T)),
\end{array}\right.
$$

where $0<a<1<b, p:[0, T]_{\mathbb{T}} \rightarrow(0, \infty)$ is right-dense continuous and $q:[0, T]_{\mathbb{T}} \rightarrow(0, \infty)$ is continuous such that

$$
\max _{t \in[0, T]_{\mathbb{T}}} \frac{q(t)}{p(t)}<\delta\left(1-m B a^{+}\right) \sup _{x \in(0, \infty)} \frac{x}{x^{a}+x^{b}}
$$

holds, where $a^{+}=\max \left\{a_{1}, a_{2}, \ldots, a_{m}\right\}$.

In fact, $f(t, x)=q(t)\left(x^{a}+x^{b}\right)$, it is easy to see that

$$
f_{0}=\lim _{x \rightarrow 0^{+}} \inf \frac{f(t, x)}{x}=\infty, \quad f_{\infty}=\lim _{x \rightarrow \infty} \inf \frac{f(t, x)}{x}=\infty, \quad \text { uniformly for } t \in[0, T]_{\mathbb{T}} .
$$

Set

$$
F(x)=\frac{x}{x^{a}+x^{b}}, \quad x>0,
$$

then $F(0+)=F(\infty)=0$, so there exists a $\rho>0$ such that

$$
F(\rho)=\sup _{x \in(0, \infty)} F(x) .
$$

So, for $\delta \rho \leq x \leq \rho$, we have

$$
\begin{aligned}
f(t, x) / p(t) x+B \sum_{k=1}^{m} I^{\rho}(k) & =\frac{q(t)}{p(t)}\left(\frac{x^{a}+x^{b}}{x}\right)+m B a^{+} \\
& \leq \max _{t \in[0, T]_{\mathbb{T}}} \frac{q(t)}{p(t)}\left(\frac{\rho^{a}+\rho^{b}}{\delta \rho}\right)+m B a^{+}<1 .
\end{aligned}
$$

Therefore, together with Corollary 3.2, it follows that the problem (3.2) has at least two positive solutions.

\section{Competing interests}

The author declares that she has no competing interests.

\section{Acknowledgements}

The author express her gratitude to the anonymous referee for his/her valuable suggestions. The research was supported by Natural Science Foundation of Gansu Province of China (Grant No. 1310RJYA080).

Received: 2 October 2014 Accepted: 14 January 2015 Published online: 25 February 2015

References

1. Agarwal, RP, Bohner, M: Basic calculus on time scales and some of its applications. Results Math. 35, 3-22 (1999)

2. Bohner, M, Peterson, A: Dynamic Equations on Time Scales: An Introduction with Applications. Birkhäuser, Boston (2001)

3. Bohner, M, Peterson, A: Advances in Dynamic Equations on Time Scales. Birkhäuser, Boston (2003)

4. Hilger, S: Analysis on measure chains - a unified approach to continuous and discrete calculus. Results Math. 18, 18-56 (1990) 
5. Belarbi, A, Benchohra, M, Ouahab, A: Existence results for impulsive dynamic inclusions on time scales. Electron. J. Qual. Theory Differ. Equ. 2005, 12 (2005)

6. Benchohra, M, Henderson, J, Ntouyas, SK, Ouahab, A: On first order impulsive dynamic equations on time scales. J. Differ. Equ. Appl. 6, 541-548 (2004)

7. Benchohra, M, Ntouyas, SK, Ouahab, A: Existence results for second-order boundary value problem of impulsive dynamic equations on time scales. J. Math. Anal. Appl. 296, 65-73 (2004)

8. Benchohra, M, Ntouyas, SK, Ouahab, A: Extremal solutions of second order impulsive dynamic equations on time scales. J. Math. Anal. Appl. 324, 425-434 (2006)

9. Chen, $\mathrm{HB}$, Wang, $\mathrm{HH}$ : Triple positive solutions of boundary value problems for $p$-Laplacian impulsive dynamic equations on time scales. Math. Comput. Model. 47, 917-924 (2008)

10. Geng, $F, X u, Y, Z h u, D:$ Periodic boundary value problems for first-order impulsive dynamic equations on time scales. Nonlinear Anal. 69, 4074-4087 (2008)

11. Graef, JR, Ouahab, A: Extremal solutions for nonresonance impulsive functional dynamic equations on time scales. Appl. Math. Comput. 196, 333-339 (2008)

12. Guan, W, Li, DG, Ma, SH: Nonlinear first-order periodic boundary value problems of impulsive dynamic equations on time scales. Electron. J. Differ. Equ. 2012, 198 (2012)

13. Henderson, J: Double solutions of impulsive dynamic boundary value problems on time scale. J. Differ. Equ. Appl. 8, 345-356 (2002)

14. Li, JL, Shen, JH: Existence results for second-order impulsive boundary value problems on time scales. Nonlinear Anal. 70, 1648-1655 (2009)

15. Li, YK, Shu, J: Multiple positive solutions for first-order impulsive integral boundary value problems on time scales. Bound. Value Probl. 2011, 12 (2011)

16. Liu, HB, Xiang, X: A class of the first order impulsive dynamic equations on time scales. Nonlinear Anal. 69, 2803-2811 (2008)

17. Wang, C, Li, YK, Fei, Y: Three positive periodic solutions to nonlinear neutral functional differential equations with impulses and parameters on time scales. Math. Comput. Model. 52, 1451-1462 (2010)

18. Wang, DB: Positive solutions for nonlinear first-order periodic boundary value problems of impulsive dynamic equations on time scales. Comput. Math. Appl. 56, 1496-1504 (2008)

19. Wang, DB: Periodic boundary value problems for nonlinear first-order impulsive dynamic equations on time scales. Adv. Differ. Equ. 2012, 12 (2012)

20. Wang, ZY, Weng, PX: Existence of solutions for first order PBVPs with impulses on time scales. Comput. Math. Appl. 56 , 2010-2018 (2008)

21. Zhang, HT, Li, YK: Existence of positive periodic solutions for functional differential equations with impulse effects on time scales. Commun. Nonlinear Sci. Numer. Simul. 14, 19-26 (2009)

22. Bainov, DD, Simeonov, PS: Impulsive Differential Equations: Periodic Solutions and Applications. Longman, Harlow (1993)

23. Benchohra, M, Henderson, J, Ntouyas, SK: Impulsive Differential Equations and Inclusions, vol. 2. Hindawi Publishing Corporation, New York (2006)

24. Samoilenko, AM, Perestyuk, NA: Impulsive Differential Equations. World Scientific, Singapore (1995)

25. Dai, QY, Tisdell, CC: Existence of solutions to first-order dynamic boundary value problems. Int. J. Differ. Equ. 1, 1-17 (2006)

26. Sun, JP, Li, WT: Existence of solutions to nonlinear first-order PBVPs on time scales. Nonlinear Anal. 67, 883-888 (2007)

27. Guan, W, Ma, SH, Wang, DB: Periodic boundary value problems for first order difference equations. Electron. J. Qual. Theory Differ. Equ. 2012, 52 (2012)

28. Torres, PJ: Existence of one-signed periodic solutions of some second-order differential equations via a Krasnoselskii fixed point theorem. J. Differ. Equ. 190, 643-662 (2003)

29. Guo, D, Lakshmikantham, V: Nonlinear Problems in Abstract Cones. Academic Press, New York (1988)

\section{Submit your manuscript to a SpringerOpen ${ }^{\circ}$ journal and benefit from:}

- Convenient online submission

Rigorous peer review

- Immediate publication on acceptance

- Open access: articles freely available online

- High visibility within the field

- Retaining the copyright to your article 